\title{
The MIDLINE trial - Managing intravenous devices among patients with limited vascular access or prolonged therapy: a pilot randomised control trial protocol
}

\author{
*Nicole Marsh ${ }^{1,2,3,4}$, Emily Larsen ${ }^{1,2,3}$, Julie Flynn ${ }^{3,4}$, Catherine O'Brien ${ }^{1,3}$, Tricia Kleidon ${ }^{1,3,5}$, Peter Groom', Barbara Hewer', \\ Claire M Rickard ${ }^{1,2,3}$ \\ 'Nursing \& Midwifery Research Centre, Royal Brisbane and Women's Hospital, QLD, Australia \\ ${ }^{2}$ School of Nursing and Midwifery, Griffith University, QLD, Australia \\ ${ }^{3}$ Alliance for Vascular Access Teaching and Research, Menzies Health Institute Queensland, QLD, Australia \\ ${ }^{4}$ School of Nursing, Queensland University of Technology, Kelvin Grove, QLD, Australia \\ ${ }^{5}$ Queensland Children's Hospital, South Brisbane, Queensland, Australia \\ *Corresponding author \\ Nicole Marsh, Nursing and Midwifery Research Centre, Royal Brisbane and Women's Hospital, Butterfield Street, Herston, QLD 4029, Australia \\ Email nicole.marsh@health.qld.gov.au
}

Keywords intravenous, vascular access devices, midline catheter, peripheral intravenous catheter, randomised controlled trial, phlebitis

For referencing Marsh N et al. The MIDLINE trial - Managing intravenous devices among patients with limited vascular access or prolonged therapy: a pilot randomised control trial protocol. Vascular Access 2020; 6(1):20-24.

Dol https://doi.org/10.33235/va.6.1.20-24

\begin{abstract}
Introduction Peripheral intravenous catheters (PIVCs) are essential for administration of intravenous fluids and medications. While patient need for PIVCs is high, up to $69 \%$ fail due to complications such as occlusion and infiltration. Midline catheters (MCs) are an alternative to PIVCs; they are $8-20 \mathrm{cms}$ in length and terminate at, or are distal to, the axillary vein, not in the central venous circulation. Midline catheters' rising popularity is due to concerns that patients' veins are depleted by multiple consecutive PIVCs. However, there have been no randomised controlled trials (RCTs) comparing these devices to guide practice.

Methods and analysis This single centre, parallel group, pilot RCT is designed to compare effectiveness of MCs with PIVCs for patients with difficult vascular access ( $\leq 2$ visible and palpable veins) and/or receiving peripherally compatible intravenous therapy for $\geq 5$ days. This trial will be conducted at the Royal Brisbane and Women's Hospital, Australia. The recruitment target is 70 participants per group $(n=140)$. There are three primary outcomes of interest to test: 1) Feasibility of an adequately powered RCT with pre-established criteria for eligibility, recruitment, protocol adherence and retention; 2) Insertion failure (number of PIVCs/MCs unable to be inserted); and 3) Post-insertion failure: all-cause failure. Secondary outcomes to be collected include: number of insertion attempts; time to insert; catheter dwell time; costs (staff time/equipment); patient-reported insertion pain; and individual causes of failure, e.g. phlebitis. Feasibility outcomes will be reported descriptively and analysed against pre-determined acceptability criteria. As a pilot study, statistical comparison methods will be tested. Cox regression will assess the effect of patient and treatment differences.
\end{abstract}

Ethics and trial commencement This pilot trial has ethical approval from Queensland Health (HREC/2018/QRBW/46295) and Griffith University (2018/962).

Trial registration ACTRN12619000383167

\section{Strengths and limitations of this study}

- This pilot randomised controlled trial (RCT) will assess the feasibility and adequacy of study methods for a larger, adequately powered RCT.

- This pilot trial is under-powered to detect the effectiveness of midline catheters (MCs) compared with peripheral intravenous catheters (PIVCs). 


\section{INTRODUCTION}

Peripheral intravenous catheters (PIVCs) are inserted for the administration of medications and fluids. 'Two billion are sold worldwide annually. ${ }^{2}$ While patient need for PIVCs is high, up to $69 \%$ fail due to complications such as occlusion, infiltration and phlebitis, ${ }^{3-6}$ necessitating additional PIVC insertions to complete treatment. ${ }^{7.8}$ PIVC replacements increase healthcare costs (staff time/equipment); when peripheral access is impossible, some patients will require the insertion of a central venous device, which has a higher insertion risk profile.,10 Midline catheters (MCs) are an alternative peripheral vascular access device to PIVCs, and are increasingly used internationally, but rarely in Australia. ${ }^{11}$ Midline catheters have been available since the 1950s; however, due to outdated materials, lost favour in the 1990s."

Since re-engineering with polyurethane, $M C$ use is increasing, but they are not complication-free, e.g. leaking, infection, and thrombosis..112 The increasing popularity of MCs is due, in part, to concerns that patients' veins are depleted by multiple PIVC placements. This is supported by recent observational data that found a MC successful functional dwell of 7.7-16.4 days, ${ }^{13}$ comparing starkly with the average PIVC dwell of 2.4-4.2 days, commonly shortened by complications. ${ }^{14,15}$ Thus, complications still can occur, but appear to occur later. A recent Australian nonrandomised study found MCs successfully dwelled for twice the duration of even ultrasound-inserted PIVCs." Midline catheter use could potentially result in patients needing only one device per treatment, in comparison to multiple PIVCs.

Both PIVCs and MCs are approved by manufacturers for up to 29 days' use, but to date there have been no randomised studies comparing these devices to guide healthcare practice and device selection. The objective of this pilot RCT is to test the feasibility of conducting a suitably powered RCT by assessing both the methodology and rigour of methods planned for the larger study.

\section{METHODS AND ANALYSIS}

\section{Design}

We will conduct a single-centre, parallel group, pilot RCT to compare MCs with PIVCs for patients with difficult vascular access ( $\leq 2$ visible and palpable veins) and/or expected to require peripherally compatible intravenous (IV) therapy for $\geq 5$ days.

\section{Hypotheses}

\section{Hypothesis 1}

The feasibility of conducting an adequately powered RCT will be established by meeting targets formulated a priori and based on results from previous PIVC pilot trials. 16,17 Targets are as follows:

- Eligibility: over $80 \%$ of patients screened will be eligible.

- Recruitment (consent): over $80 \%$ of eligible participants will agree to enrol.
- Protocol adherence: over $90 \%$ of participants in the intervention groups will receive their allocated treatment.

- Retention and attrition: fewer than $5 \%$ of patients will be lost to attrition.

\section{Hypothesis 2}

Patients with a MC will have fewer episodes of insertion failure (unable to be successfully placed) compared to those with a PIVC.

\section{Hypothesis 3}

Patients with a MC will have fewer episodes of all-cause post-insertion device failure: a composite of pain, infiltration/ extravasation, blockage/occlusion (with or without leakage), phlebitis, thrombosis, dislodgement (complete or partial), or infection (laboratory-confirmed local or bloodstream infection $[B S I])$, compared to those with a PIVC.

\section{Setting}

The pilot RCT will be conducted in a single-centre, referral teaching hospital, the largest provider of healthcare services in Queensland, Australia, with just under 1000 beds.

\section{Ethics}

This study has obtained approval from the hospital Human Research Ethics Committee (HREC) (HREC/2018/QRBW/46295) and Griffith University (2018/962). Written informed consent will be obtained from all study participants prior to randomisation. Data will be stored securely in a password-protected database and paper copies in a locked filing cabinet, as per the Australian National Health and Medical Research Council guidelines.

\section{Participants}

Participants for this RCT will be patients admitted to general medical or surgical wards. They are eligible for inclusion if: equal to or over the age of 18; able to provide informed consent; have difficult vascular access ( $\leq 2$ palpable veins); and/or are expected to require $\geq 5$ days of peripherally compatible IV therapy. They will be excluded from recruitment if: they have a current BSI (within 24 hours); they have a co-existent central venous access device (CVAD); are non-English speaking without an interpreter; receiving end-of-life care; have a cognitive barrier to consent (without a substitute decision maker); or have been previously enrolled in the study.

\section{Sample size}

This pilot RCT has a recruitment target of 140 participants, 65 patients per arm plus five per arm for potential attrition. As this is a feasibility trial, our sample size is not determined by statistical power to test hypotheses, rather to test protocol feasibility and gain initial estimates of effect.18,19 As recruitment is in a large referral hospital with just under 1000 beds, purchasing in excess of 200,000 PIVCs per year, we expect a high number of eligible patients. 


\section{Interventions}

The control group of this trial will have PIVCs inserted: in line with hospital policy by an accredited PIVC inserter; at the bedside; using landmark/palpation and, aseptic non-touch technique. The PIVCs will be BD Insyte ${ }^{\mathrm{TM}}$ Autoguard ${ }^{\mathrm{TM}} \mathrm{BC}$.

For the intervention group the $M C$ insertions will be placed by a registered nurse with established skills, at the bedside or in a dedicated procedure room (depending on suitability of environment), using ultrasound and a surgical aseptic non-touch technique. Midlines will be PowerGlide Pro ${ }^{\mathrm{TM}}$ Midline Catheters.

\section{OUTCOME MEASURES AND DEFINTIONS}

\section{Feasibility outcomes}

The feasibility of conducting an adequately powered RCT will be assessed against the following criteria: 1. Eligibility (percentage of eligible screened patients); 2. Recruitment (percentage of eligible patients who consent to trial participation); 3. Protocol adherence (percentage of patients who receive the allocated intervention); and 4. Retention and attrition (percentage of lost to follow-up).

\section{Primary outcomes}

- Insertion failure: Proportion of PIVCs/MCs that are unable to be successfully inserted (measured from the time of randomisation until 24 hours post-randomisation).

- Post-insertion failure: All-cause post-insertion failure: a composite of pain, infiltration/extravasation, blockage/ occlusion (with or without leakage), phlebitis, thrombosis, dislodgement (complete or partial) or infection (laboratoryconfirmed local or BSI). This composite measure incorporates the multifocal path to the same endpoint of PIVC failure.

\section{Secondary outcomes}

- Number of insertion attempts (needle punctures to insert device), documented by inserter (or observed by the research nurse $(\operatorname{ReN}))$.

- Time to insert device (from randomisation to successful insertion).

- Device dwell-time (time from insertion to removal, in hours).

- Patient-reported pain of insertion procedure (0-10 verbal rating scale).

- Patient-reported satisfaction regarding insertion procedure (0-10 verbal rating scale).

- Serious adverse events (e.g. intensive care unit admission), and adverse events (e.g. insertion site itch or rash, haematoma).

- Cost (cost and number of products used, cost of treating complications, staff time for device insertion).

- Infection (primary BSI): Centers for Disease Control (CDC), National Health and Safety Network criteria $(\mathrm{NHSN})^{20}$.
Bloodstream infection is defined as positive blood culture from a peripheral vein; clinical signs of infection (e.g. fever, chills, or hypotension and a colonised IV catheter tip culture ( $\geq 15$ colony forming units) with the same organism as identified in the blood; or purulent drainage from the involved vascular site; and no other apparent source for the BSI except the IV catheter (in situ within 48 hours of the BSI).

- Blockage/occlusion: the device will not infuse, with or without leakage, out of the entry site when fluid is infused.

- Infiltration/extravasation: the movement of IV fluid into surrounding tissue (infiltration), with or without resulting tissue damage (extravasation).

- Dislodgement: either partial or complete.

- Phlebitis: two or more signs/symptoms of: pain/tenderness; redness; swelling; or palpable cord/vein streak from the entry site.

- Thrombosis (either suspected or confirmed): suspected - as assessed/suspected by the treating clinician; confirmed ultrasound/venographic confirmed thrombosed vessel at the device site.

- $\quad$ Pain (patient-reported), resulting from the device dwell.

- Subsequent device required (until discharge, insertion of CVAD or no PIVC/MC in situ for 48 hours).

\section{STUDY PROCEDURES}

\section{Recruitment, randomisation, allocation concealment and blinding}

A ReN will screen the wards daily to identify patients who are eligible for inclusion. They will liaise with the treating team (nurses and medical staff as appropriate) to gauge the duration of time patients are expected to require IV treatment. If patients are potentially eligible, the bedside nurse will ask their permission to be approached about the trial. If they agree, the ReN will explain the trial, answer questions, and provide a written patient information document. Patients will be given ample time to consider participation; those that agree to participate will sign the consent form to enter the trial.

Using Griffith University Clinical Trials Randomisation Service, with allocation concealment, patients will be randomised (varied block sizes and 1:1 ratio) to either the PIVC or MC group. A unique study ID will also be assigned. Blinding of patients/clinicians to the intervention is not possible; however, the primary endpoint BSI will be assessed by a blinded infectious diseases expert using the CDC NHSN criteria. ${ }^{20}$ In addition, the statistician will be blinded to group allocation for data analysis.

\section{Other aspects of PIVC/MC care}

In both groups, local hospital policies will be followed, including: chlorhexidine in alcohol skin decontamination prior to insertion; clipping of hair (if present); use of sterile transparent dressings and 
securement; and needleless connector, including attachments. Local anaesthetic (lignocaine hydrochloride) will also be offered as per hospital policy. Catheter diameter and length will be chosen by the inserter to match the vessel. Devices will be assessed each shift by clinical staff and documented as usual. Devices will be removed by the patients' treating clinicians as per usual clinical practice, for example, if the device is no longer needed, if suspected of infection, is painful, the site is swollen, or the device is dysfunctional (leaks, dislodges or occludes).

\section{Strategies to promote protocol adherence}

To promote adherence to the study protocol, any clinical staff caring for participants on this study will be provided with education about the study protocol prior to the RCT commencement and during the trial. The researcher will be available to answer any clinician queries throughout the study.

\section{Data collection}

The ReNs will collect patient demographic and device characteristics and perform daily site assessments for complications such as redness or pain. Data will be collected from the patients' medical records, using hand-held devices and entered into the REDCap database (Research Electronic Data CAPture, Vanderbilt). ${ }^{21}$ Data will be entered using a unique study ID (no participant identifiers entered), only re-identifiable using a separate screening log, which will be kept in a secure location on-site (for later secure archiving). This REDCap database will require a valid username and password, only provided to ReNs and principal investigators based at the recruiting site.

The trial co-ordinator/project manager will undertake quality checks for allocation integrity and monitor 100\% source data verification for: the first five patients; consent forms; primary outcomes; and a random 5\% of other data for all patients. Upon trial completion, only the statistician, trial co-ordinator and chief/ principal investigators will have access to the de-identified data (once exported).

At enrolment: ReNs will collect data on: patient demographics (e.g. age, gender, weight); clinical factors (e.g. general diagnosis, co-morbidities, current infection/s); device factors (device allocation, insertion site, inserter discipline, technology assisted insertion, number of insertion attempts, size/gauge, side [right/ left]); and insertion department. Patients will be surveyed to assess their reported pain on insertion (0-10 verbal numerical rating scale).

Daily checks: The ReN will visit patients daily to document the presence and condition of the allocated device and assess the insertion site for complications (e.g. redness, discharge, pain, swelling); reinforce the protocol with patients and nurses; and assess for primary, secondary and adverse outcomes.
Device removal: At device removal, the ReN will record the reason for removal, document treatment received (e.g. intravenous antibiotics (IVABs), other infusates), conduct a site assessment (for device/site complications), and collect data from electronic records. Discharged patients (where it is unclear if/why the device was removed) will have follow-up data collected by telephone (limited to two attempts if unanswered). Alternatively, patients can contact study staff at any time if they have any questions or concerns (contact details available on the Participant Information Sheet and Consent Form). Patient-reported satisfaction (overall) will be assessed on a verbal numerical rating scale (0-10).

\section{Statistical analysis}

Feasibility outcomes will be reported descriptively and analysed against pre-determined acceptability criteria, e.g. $<5 \%$ attrition. Data will be exported into IBM SPSS Statistics version 22 (SPSS) for analysis. An intention-to-treat analysis framework will be used; the unit of analysis will be one PIVC/MC per patient. Numbers (\%) will be reported for categorical data. Mean values and standard deviations (SD) will be reported for normally distributed data; median values and $25 \mathrm{th} / 75$ th percentiles reported otherwise. As a pilot for a superiority RCT, the following statistical comparison methods will be piloted. Cox regression will assess the effect of patient and treatment differences as well as group comparisons of post-insertion device failure ( $p<0.05$ significant). A graph of the Kaplan-Meier survival function will be generated, and the proportional hazards assumption checked with the log-log plot of survival, and log-rank test performed.

Costs will be estimated by assessing cost and number of products used (whole sample) and staff number and length of time required (e.g. minutes) for device insertion (a sub-set of 10 participants per group, convenience sample). Total cost $=$ clinician (directly measured time $\mathrm{x}$ estimated hourly salary) + fixed cost (supplies).

\section{Validity and reliability}

Strategies employed to maintain internal validity for this trial include: web-based randomisation and allocation concealment until randomisation; blinding of the study statistician, infectious diseases expert; and an intention-to-treat approach, all patients randomised will be accounted for. External validity for this study will be improved by clearly defining the characteristics of the target population and inclusion and exclusion criteria.

Reliability will be assessed by inter-rater reliability testing for 5\% of PIVC site daily inspections and outcome assessment between the daily assessor and an independent vascular access expert.

\section{DISCUSSION}

This pilot RCT will be the first to compare the possible benefits of MCs for patients with difficult vascular access or requiring prolonged IV therapy. As the trial is being conducted in the medical and surgical wards of a large metropolitan tertiary 
hospital, the results will be generalisable to most general ward patients with difficult vascular access. This trial will test the feasibility and safety of the study protocol for a larger definitive RCT. In addition, the results from this trial will aid the development of $\mathrm{MC}$ education and provide preliminary evidence to guide local and international clinical guidelines about the use of MCs.

\section{DISCLOSURES}

Nicole Marsh's previous employer, Griffith University, has received, on her behalf, investigator-initiated research grants and unrestricted educational grants from Becton Dickinson and Cardinal Health, and a consultancy payment provided to Griffith University from Becton Dickinson for clinical feedback related to vascular access device placement and maintenance (unrelated to the current project).

Emily Larsen's employer, Griffith University, has an investigatorinitiated research grant from Cardinal Health (formerly Medtronic), and a conference scholarship attendance supported by Angiodynamics (unrelated to the submitted work) to support her research.

Tricia Kleidon reports investigator-initiated research grants and speaker fees provided to Griffith University from 3M Medical, Angiodynamics, Baxter, Becton Dickinson, Centurion Medical, Cook Medical, Medical Specialties Australia, Smiths Medical and Vygon (unrelated to the current project).

Claire Rickard's employer Griffith University has received unrestricted investigator-initiated research or educational grants on her behalf from product manufacturers (3M, Adhezion, Angiodynamics, Baxter, BD-Bard, Cardinal Health, Centurion Medical Products, Eloquest, Medtronic, Smiths Medical) and consultancy payments from manufacturers (3M, BBraun, BD-Bard, ResQDevices, Smiths Medical).

Julie Flynn, Catherine O'Brien, Peter Groom and Barbara Hewer have no disclosures.

\section{REFERENCES}

1. Chopra V, Flanders SA, Saint S, Woller SC, O'Grady NP, Safdar N, et al. The Michigan Appropriateness Guide for Intravenous Catheters (MAGIC): results from a multispecialty panel using the RAND/UCLA appropriateness method. Ann Intern Med. 2015;163(6_Supplement):S1-S40.

2. Rickard CM, Ray-Barruel G. Peripheral intravenous catheter assessment: beyond phlebitis. Lancet Haematol. 2017;4(9):e402-e403.

3. Marsh N,Webster J, Larson E, Cooke M, Mihala G, Rickard CM. Observational study of peripheral intravenous catheter outcomes in adult hospitalized patients: a multivariable analysis of peripheral intravenous catheter failure. J Hosp Med. 2018:E1-E7.

4. Rickard CM, Marsh N, Webster J, Runnegar N, Larsen E, McGrail, M, et al. Dressings and securements for the prevention of peripheral intravenous catheter failure in adults (SAVE): a pragmatic, randomised controlled, superiority trial. Lancet. 2018;392(10145):419-430.

5. Dillon MF, Curran J, Martos R, Walsh C, Walsh J, Al-Azawi SE, et al. Factors that affect longevity of intravenous cannulas: a prospective study. QJM. 2008;101(9):731-735.
6. Marsh N, Webster J, Flynn J, Mihala G, Hewer B, Fraser J, et al. Securement methods for peripheral venous catheters to prevent failure: a randomised controlled pilot trial. JVA. 2015;16(3):237-244.

7. Royer TF. Improving short peripheral IV outcomes: a clinical trial of two securment methods. JVA. 2003;8(4):45-49.

8. Smith B. Peripheral intravenous catheter dwell times: a comparison of 3 securement methods for implementation of a 96-hour scheduled change protocol. J Infus Nurs. 2006;29(1):14-17.

9. Marsh N, Webster J, Mihala G, Rickard CM. Devices and dressings to secure peripheral venous catheters to prevent complications. Cochrane Libr. 2015(6).

10. Monreal M, Quilez F, Rey-Joly C, Rodriguez S, Sopena N, Neira, C, et al. Infusion phlebitis in patients with acute pneumonia: a prospective study. Chest. 1999;115(6):1576-1580.

11. Alexandrou E, Mifflin N, Craig M, Sou V, Frost SA. Extended dwell peripheral catheters in patients with difficult venous access: Comparison of a peripheral intravenous catheter and midline catheter. Vascular Access. 2018;4(1).

12. Anderson NR. Midline catheters: the middle ground of intravenous therapy administration. J Infus Nurs. 2004;27(5):313-321.

13. Adams DZ, Little A, Vinsant $C$, Khandelwal S. The midline catheter: A clinical review. J Emerg Med. 2016;51(3):252-258.

14. Rickard CM, Webster J, Wallis MC, Marsh N, McGrail MR, French V, et al. Routine versus clinically indicated replacement of peripheral intravenous catheters: a randomised controlled equivalence trial. Lancet. 2012;380(9847):1066-1074.

15. DeVries $M$, Valentine $M$, Mancos $P$. Protected clinical indication of peripheral intravenous lines: successful implementation. JAVA 2016;21(2):89-92.

16. Marsh N, Webster J, Larsen E, Genzel J, Cooke M, Mihala G, et al. Expert versus generalist inserters for peripheral intravenous catheter insertion: a pilot randomised controlled trial. Trials. 2018;19(1):564.

17. Keogh S, Flynn J, Marsh N, Mihala G, Davies K, Rickard CM. Flushing in Peripheral Catheters: The FliP Trial. A pilot, factorial randomized controlled trial of high and low flushing frequencies and volumes. JVA (in progress). 2015.

18. Hertzog MA. Considerations in determining sample size for pilot studies. Research Nurs Health. 2008;31(2):180-191.

19. Julious SA. Sample size of 12 per group rule of thumb for a pilot study. Pharm Stat. 2005;4(4):287-291.

20. NHSN, National Healthcare Safety Network (NHSN) Patient Safety Component Manual, CDC, Editor. 2018: Atlanta. p. 1-38.

21. Harris PA, Taylor R, Thielke R, Payne J, Gonzalez N, Conde JG. Research electronic data capture (REDCap)-a metadata-driven methodology and workflow process for providing translational research informatics support. J Biomed Inform. 2009;42(2):377-381. 